\title{
Rapid and Progressive CD4+ Decline in a Monkey Infected with an SIV/HIV-1 Chimeric Virus
}

Daiji NAGAMACHI, Tatsuhiko IGARASHI'), Toshihide SHIMADA, Takeo KUWATA ${ }^{1)}$, Masahiro UI ${ }^{1)}$, Yasushi AMI'), Yoshimi ENOSE'), Eiji IDO'), Manabu FUKUMOTO ${ }^{3)}$ and Masanori HAYAMI)*

Department of Pathology and Tumor Biology, Graduate School of Medicine and ${ }^{11}$ Institute for Virus Research, Kyoto University, Kyoto 606, ${ }^{2}$ National Institute of Infectious Disease, Japan, Tokyo 208, and ${ }^{3)}$ Department of Pathology and Biology of Diseases, Graduate School of Medicine, Kyoto University, Kyoto 606, Japan

(Received 4 September 1997/Accepted 20 October 1997)

ABSTRACT. We previously constructed a simian immunodeficiency virus/human immunodeficiency virus type 1 (HIV-1) chimeric virus, NM-3rN to generate a pathogenic HIV-1 in macaque monkeys. During the in vivo passage of this virus in several monkeys, a viral strain, R43-56 was obtained which acquired a better replication ability in vivo. MM121, one of the three monkeys inoculated with the R43-56, showed weight loss, diarrhea and a rapid and continuous decrease in $\mathrm{CD} 4^{+}$lymphocytes at the moribund stage. An autopsy revealed generalized lymphadenopathy, dehydration, and ileocecal intussusception. In situ hybridization showed that the virus infection was in systemic lymphoid organs. We are presently monitoring the survivors to obtain candidates for a more virulent virus. R43-56 may be a better challenge virus and useful tool for human acquired immunodeficiency syndrome research. — KEY wORDS: challenge virus, HIV-1 vaccine development, SIV/HIV-1 chimeric virus.

J. Vet. Med. Sci. 60(3): 361-363, 1998

To establish disease prevention and develop therapies against acquired immune deficiency syndrome (AIDS), development of an animal model for human immunodeficiency virus type 1 (HIV-1) infection is important. However, even a susceptible animal model, the chimpanzee, is limited by its low availability.

To overcome this problem, we attempted to generate an HIV-1 that was pathogenic in macaque monkeys. We previously constructed SIV/HIV-1 chimeric viruses based on simian immunodeficiency virus (SIV) which contained SIV genes responsible for monkey cell tropism [5]. The original chimeric virus (NM-3rN [4]) had vpr, env, tat, rev and vpu of HIV-1(NL432 [1]) and other genes derived from SIVmac (MA239 [3]). The virus was subjected to in vivopassage in several rhesus macaques. A virus strain (R43-56) was recovered at 56 weeks post inoculation (w.p.i.) from one of the monkeys (MM43) which exhibited a high virus load [4]. R43-56 acquired improved replication ability in monkey peripheral blood mononuclear cells (PBMC) as compared with the original NM-3rN (Fig. 1). The recovered virus was then inoculated intravenously into three juvenile rhesus monkeys (MM104, MM106 and MM121) free of simian retrovirus type D and simian T-cell leukemia virus type 1 . Two to 12 w.p.i., these monkeys showed a 10- to 50 -fold higher level of cell-associated virus loads as compared with NM-3rN (for example, $>2.1 \times 10^{3}$ cells $/ 10^{6}$ PBMC of MM121, $1.0 \times 10^{2}$ cells $/ 10^{6} \mathrm{PBMC}$ of an NM$3 \mathrm{rN}$-infected monkey (MM050)) and antibodies against several viral proteins (Fig. 2). Among these monkeys, MM121, the number of $\mathrm{CD}^{+}$lymphocytes started to decline at 1 w.p.i. followed by a continuous decrease until the

* Correspondence to: Hayami, M., Laboratory of Pathologic Viruses, Institute for Virus Research, Kyoto University, Sakyo-ku, Kyoto 606, Japan. moribund stage (Fig. 3). The number of $\mathrm{CD}^{+}$cells dropped at 1 w.p.i., recovered temporarily, and again diminished. A gradual loss of body weight was also observed after 7 w.p.i.. As the monkey showed a combination of severe diarrhea with rapid weight loss at 14 w.p.i., it was euthanized and autopsied.

Macroscopically, generalized lymphadenopathy, intussusception of ileocecal region and dehydration were observed. A histopathological examination of lymph nodes revealed fibrosis of the germinal center and an expanded

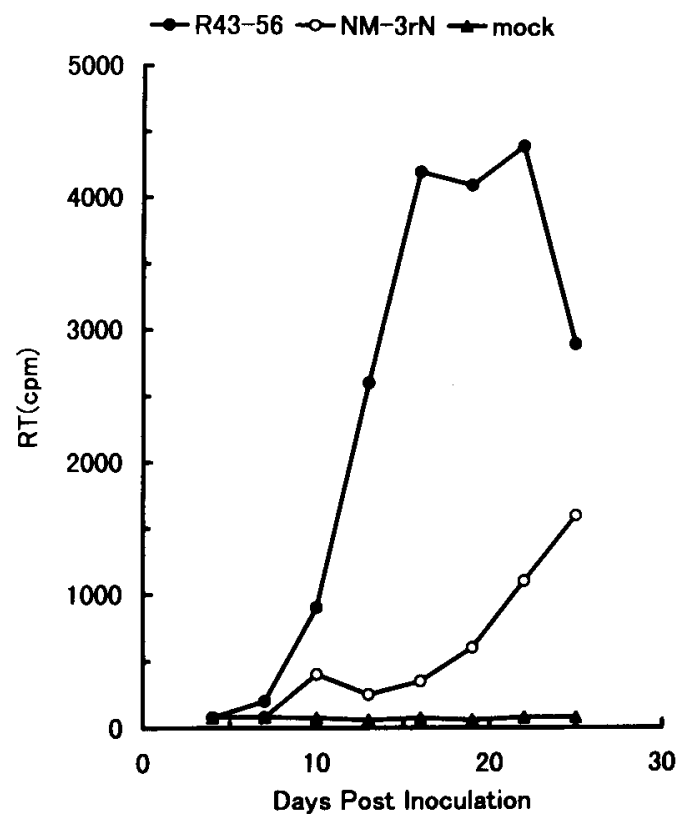

Fig. 1. R43-56 acquired more competent replicative capacity than the original NM$3 \mathrm{rN}$ in monkey PBMCs. 
paracortex. The viral RNA was identified in germinal center cells by an RNA-RNA in situ hybridization technique as reported in AIDS-like disease in SIV-infected rhesus monkeys (Fig. 4) [2]. Ileocecal intussusception was doublebarreled and associated with intramural hemorrhagic necrosis and severe enteric trichuriasis. Even though there was no evidence of extra-enteric parasitosis, these lesions seemed to be responsible for the severe diarrhea and impairment at the late stage. The monkey also showed

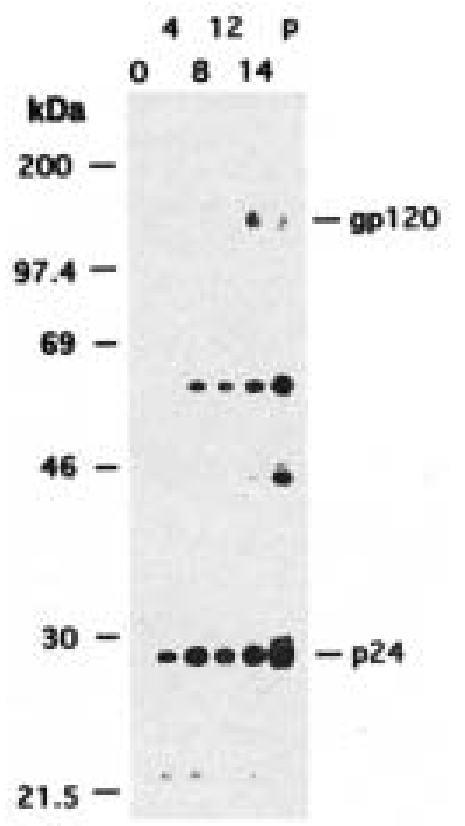

Fig. 2. Antibody response of a monkey infected with the R43-56 determined by Western blotting analysis. Plasma samples collected at intervals were treated with SDS-PAGE-resolved antigens of parental HIV-1 (NL432) after transfer to membranes. The numbers of weeks after inoculation are shown above. The serum from a monkey infected with the NM-3rN was tested as a positive control $(\mathrm{P})$. Size markers are indicated on the left.

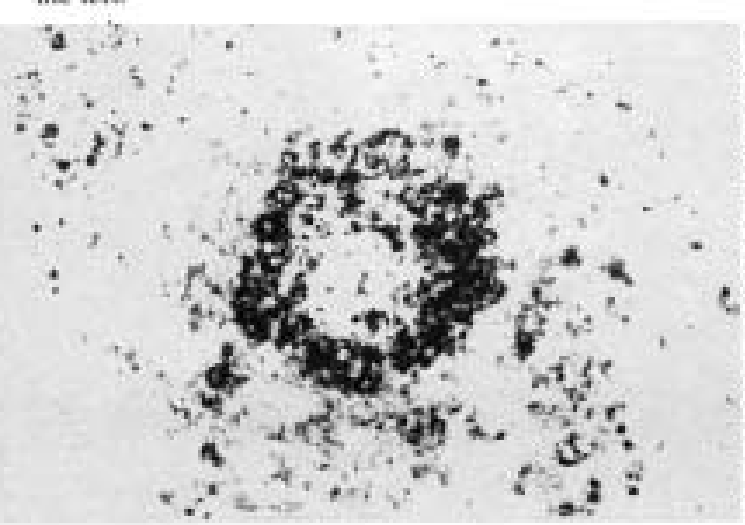

acute hepatitis with acidophilic bodies in hepatocytes that might reveal the presumed etiology of the viral infection. Besides these findings, there was no significant opportunistic infection in the respiratory tract or other systems.

We previously reported that the NM-3rN which possessed HIV-1 Env enabled the evaluation HIV-1 Env-targeted vaccine candidates by using macaque monkeys instead of chimpanzees [4]. The virus obtained in this study (R4356), which caused a persistent viral infection and higher replication rate in vivo might be a better challenge virus than the original NM-3rN. One of the R43-56-infected macaques, MM121, showed a continuous $\mathrm{CD}^{+}{ }^{+}$decline at the early stage of infection and suddenly developed intussusception associated with trichuriasis. Even though

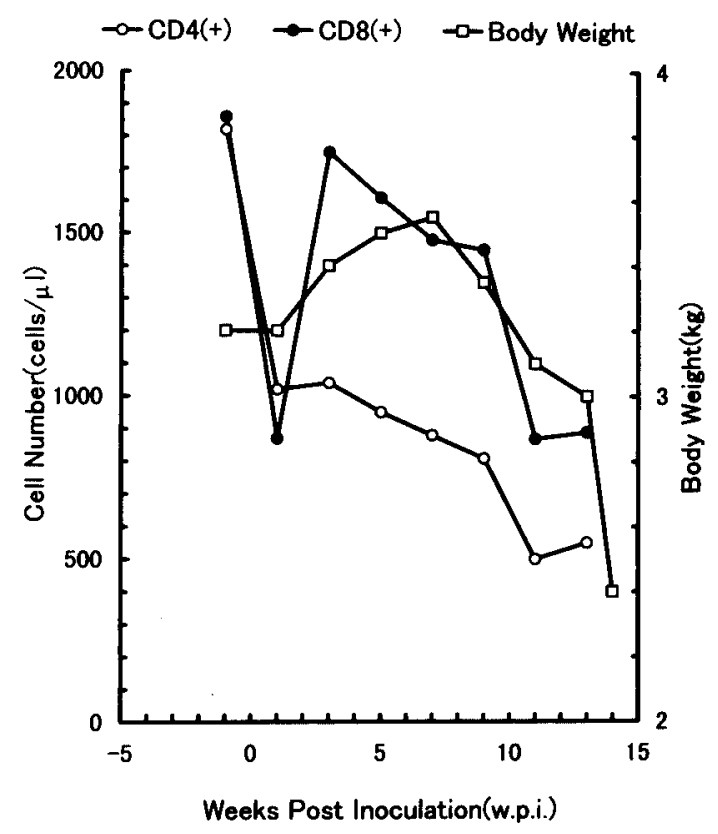

Fig. 3. Change of peripheral $\mathrm{CD}^{+}$and $\mathrm{CD} 8^{+}$ lymphocyte subsets and body weight in a monkey infected with R43-56.

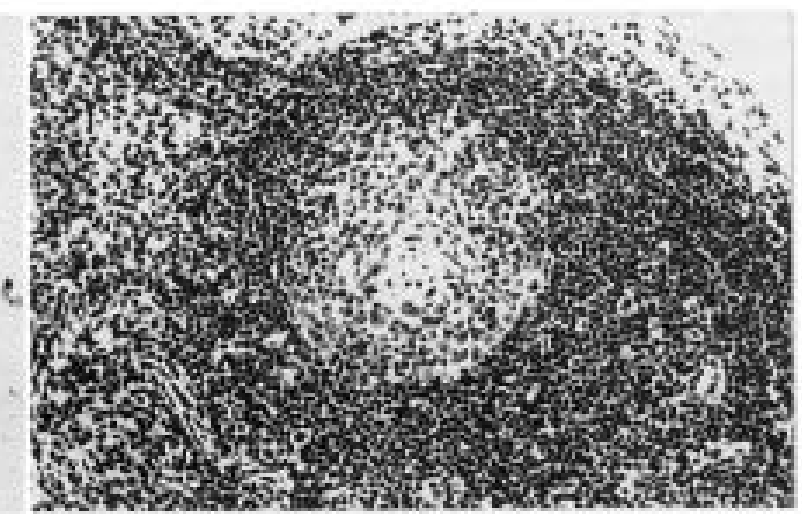

Fig. 4. RNA-RNA in situ hybridization using digoxigenin labeled riboprobe against the SIV nef-LTR region. The hybridization signals around the germinal center are detected as black dots $(\times 200$, left). The right panel shows the corresponding germinal center stained with H\&E. 
the evaluation of this parasitic infection is difficult and the symptoms do not fulfill the AIDS-criteria, the evidences of in vivo replication and rapid and progressive $\mathrm{CD} 4^{+}$decline might mean increased virulence of the virus. Based on these findings, we are continuing to monitor the survivors, MM104 and 106, and to perform the serial passage of R4356 substrain derived from the MM121. Exploring the development of pathogenicity in the R43-56 substrain enables to elucidate the roles of HIV-1 gene products in in vivo pathogenesis. Moreover, this virus will be used for evaluation of drugs (e.g., antisense oligonucleotides or antiTat compounds) directed against HIV-1 tat or rev, and pathogenic SIV/HIV-1 chimeric viruses will provide new tools for AIDS research.

\section{REFERENCES}

1. Adachi, A., Gendelman, H. E., Koenig, S., Folks, T., Willey, R., Rabson, A. and Martin, M. A. 1986. J. Virol. 59: 284291.

2. Chakrabarti, L., Isola, P., Cumont, M. C., Claessens-Maire, M. C., Hurtrel, M., Montagnier, L. and Hurtrel, B. 1994. Am. J. Pathol. 144: 1226-1237.

3. Kestler, H., Kodama, T., Ringler, D., Marthas, M., Pedersen, N., Lackner, A., Regier, D., Sehgal, P., Daniel, M., King, N. and Desrosiers, R. 1990. Science 248: 1109-1111.1.

4. Kuwata, T., Igarashi, T., Ido, E., Jin, M., Mizuno, A., Chen, J. and Hayami, M. 1995. J. Gen. Virol. 76: 2181-2191.

5. Shibata, R., Kawamura, M., Sakai, H., Hayami, M., Ishimoto, A. and Adachi, A. 1991. J. Virol. 65: 3514-3520. 